\title{
Avaliação dos mecanismos resistentes ao cisalhamento em vigas de concreto armado
}

\author{
Evaluation of shear resistant mechanisms in reinforced concrete beams
}

\author{
Iva Emanuelly Pereira Lima ${ }^{1 *}$, Vitor Bruno Santos Pereira ${ }^{2}$, Vinicius Costa Correia ${ }^{3}$
}

\begin{abstract}
RESUMO
Na elaboração de um projeto estrutural de concreto armado, uma etapa muito importante é o cálculo das armaduras transversais em vigas, que têm como principal função combater os esforços de cisalhamento. A partir disso, o presente trabalho tem como objetivo realizar um estudo comparativo entre os modelos de cálculo I e II, dispostos na ABNT NBR 6118 (2014), que determina a armadura transversal de elementos em concreto armado. Para isso, fez-se esta análise para duas seções transversais, que foram submetidas às forças cortantes correspondentes a $V_{k, 1}=100 \mathrm{kN}, V_{k, 2}=150 \mathrm{kNe} V_{k, 3}=200 \mathrm{kN}$. Ademais, para o modelo de cálculo I, foi fixado o valor de $\theta=45^{\circ}$ e, para o modelo de cálculo II, o valor de $\theta$ variou de $30^{\circ}$ a $45^{\circ}$. A partir disso, verificou-se que, de forma geral, em relação ao modelo de cálculo II, à medida que se aumenta o valor de $\theta$, para a mesma intensidade de força solicitante de cálculo, o valor da área de aço para armadura transversal também aumenta. Ademais, o modelo II se mostrou mais econômico que o modelo I para fissuras com inclinação entre $30^{\circ}$ e $35^{\circ}$, no entanto, para fissuras com inclinação igual a $45^{\circ}$, o modelo I se mostrou mais econômico.
\end{abstract}

Palavras-chave: Análise comparativa; Vigas; Cisalhamento; Armadura transversal.

\begin{abstract}
In the elaboration of a reinforced concrete structural project, a very important step is the calculation of the transversal reinforcement in beams, whose main function is to combat the shear forces. From this, the present work aims to carry out a comparative study between calculation models I and II, provided in ABNT NBR 6118 (2014), which determines the transverse reinforcement of reinforced concrete elements. For this, this analysis was performed for two cross sections, which were subjected to shear forces corresponding to $V_{k, 1}=100 \mathrm{kN}, V_{k, 2}=150 \mathrm{kN}$ and $V_{k, 3}=200 \mathrm{kN}$. Furthermore, for calculation model I, the value of $\theta=45^{\circ}$ was fixed, and for calculation model II, the value of $\theta$ ranged from $30^{\circ}$ to $45^{\circ}$. From this, it was found that, in general, in relation to calculation model II, as the value of $\theta$ is increased, for the same design load intensity, the value of the steel area for transverse reinforcement it also increases.
\end{abstract}

\footnotetext{
${ }^{1}$ Universidade Federal de Alagoas. *E-mail: ivaemanuellyl@gmail.com

${ }^{2}$ Universidade Federal do Vale do São Francisco

${ }^{3}$ Universidade Federal de Alagoas
} 
Furthermore, model II proved to be more economical than model I for cracks with an inclination between $30^{\circ}$ and $35^{\circ}$, however, for cracks with an inclination equal to $45^{\circ}$, model I was more economical.

Keywords: Comparative analysis; Beams; Shearing; Transverse reinforcement.

\section{INTRODUÇÃO}

O cálculo de uma estrutura deve ser realizado de forma a garantir que ela suporte todas as solicitações a que ela está sendo submetida e, para isso, deve-se fazer uma análise estrutural que melhor represente o comportamento do elemento estudado, considerando como fator de interferência a sua estabilidade e segurança (ARAÚJO, 2001; SADD, 2009; HIBBELER, 2010). De acordo com Carvalho e Figueiredo Filho (2016), um dos objetivos da análise estrutural é permitir, com segurança adequada, que a estrutura mantenha certas características que possibilitem a utilização satisfatória da construção durante toda a sua vida útil.

A partir disso, uma das aplicações desse tipo de análise é na elaboração de um projeto estrutural, que compreende todo o dimensionamento dos elementos estruturais que constituem uma edificação, os quais são caracterizados, principalmente, pelas lajes, vigas, pilares e fundações (ALMEIDA, 2004; FUSCO, 2000; BASTOS, 2015). Dessa forma, na elaboração de um projeto estrutural de concreto armado, uma etapa muito importante é o cálculo das armaduras transversais em vigas, que têm como principal função combater os esforços de cisalhamento (BRANDÃO, 1998; ARAÚJO, 2010; fib, 2013; BASTOS, 2015).

Sendo assim, o dimensionamento incorreto e/ou indevido de mecanismos que combatam esses esforços pode causar, por exemplo, fissuras de cisalhamento nas vigas, que normalmente acontecem nos pontos de cortante máximo e podem ser causadas tanto pela falta de armadura transversal quanto pela determinação incorreta desse tipo de armadura (MARCELLI, 2007; CARVALHO, 2016; VITOR; SANTOS; TRAUTWEIN, 2018). Ademais, as fissuras consistem em um tipo de patologia que podem intervir na estética, na durabilidade e nas particularidades estruturais de uma edificação, então, métodos que previnam e minimizem esse tipo de patologia, devem ser aplicados para um 
dimensionamento adequado da estrutura (MELLER \& VARGAS, 2016; COELHO NETO, 2017; ISHIHARA, 2017).

Em síntese, o dimensionamento das armaduras transversais em vigas de concreto armado tem como principal função combater os esforços de cisalhamento que são provenientes de esforços cortantes (PINHEIRO; MUZARDO; SANTOS, 2003; BASTOS, 2017). A partir disso, a ABNT NBR 6118 (2014) recomenda dois modelos de cálculo (modelo I e II) para a determinação dessa armadura, onde esses modelos têm um comportamento análogo ao de uma treliça de banzos paralelos e, que segundo Bastos (2015) e Pinheiro (2007), são baseados na analogia entre uma viga fissurada e uma treliça, que simbolizam a armadura transversal como as diagonais inclinadas tracionadas.

Conforme a ABNT NBR 6118 (2014), o modelo de cálculo I admite diagonais de compressão inclinadas de $\theta=45^{\circ}$ em relação ao eixo longitudinal do elemento estrutural e, admite ainda, que a parcela complementar $V_{c}$ (força cortante absorvida por mecanismos complementares ao de treliça) tenha valor constante independente de $V_{s d}$ (força cortante solicitante de cálculo). Isto significa que esse modelo tem o valor de $\theta$ fixo e o valor da força absorvida por mecanismos complementares da treliça independe do valor da força solicitante.

Em relação ao modelo II, ainda de acordo com a ABNT NBR 6118 (2014), considera-se que o valor de $\theta$ possa variar livremente entre o intervalo de $30^{\circ}$ e $45^{\circ}$ em relação ao eixo longitudinal e que a parcela complementar $V_{c}$ sofre redução à medida que o valor de $V_{s d}$ aumenta. Isto representa que para cada valor de $\theta$ escolhido, em conformidade com o intervalo mencionado, encontra-se uma armadura com um valor diferente, além do valor $V_{c}$ depender diretamente do $V_{s d}$.

Como pode ser visto, apesar dos dois modelos serem baseados na mesma hipótese, eles apresentam diferentes condições de cálculo em virtude das diferentes considerações adotadas e, por consequência, podem apresentar valores distintos de armaduras transversais. Dessa forma, pode-se fazer uma análise comparativa entre os modelos de cálculo I e II a fim de verificar o procedimento que apresenta uma melhor viabilidade econômica, analisando os valores calculados de área de aço pelos dois métodos avaliados.

A partir dessa problemática, o presente trabalho tem como objetivo realizar um estudo comparativo entre os dois modelos de cálculo, analisando as possíveis diferenças encontradas na área de aço calculada para, então, determinar e avaliar o modelo de cálculo 
mais econômico. Para isso, fez-se esta análise para duas seções transversais correspondentes a $20 \times 60 \mathrm{~cm}$ e $20 \times 70 \mathrm{~cm}$ e, em conformidade com o modelo de cálculo I, o valor do ângulo foi fixado e equivale a $\theta=45^{\circ}$ e, para o modelo II, os ângulos utilizados correspondem a $\theta=30^{\circ}, \theta=35^{\circ}$ e $\theta=45^{\circ}$.

\section{METODOLOGIA}

O objeto de estudo se localiza em uma residência unifamiliar situada na cidade de Delmiro Gouveia - AL que, segundo dados do IBGE (2017), possui uma população estimada de 52.597 habitantes, conta com uma área territorial de $626.690 \mathrm{~km}^{2}$ e a cidade é pertencente ao interior de Alagoas. A partir disso, ao que refere-se a coleta de dados, em conformidade com as características da localidade, para o cálculo das armaduras transversais, foram utilizados os seguintes dados de projeto:

- Aço CA50;

- Classe de agressividade ambiental II;

- Resistência característica do concreto a compressão de $25 \mathrm{MPa}$;

- Seção transversal I: 20 x $60 \mathrm{~cm}$;

- Seção transversal II: 20 x $70 \mathrm{~cm}$;

- Diâmetro das barras longitudinais: $8,0 \mathrm{~mm}$;

- Diâmetro das barras transversais: $6,3 \mathrm{~mm}$;

- $\propto=90^{\circ}$ (estribos verticais);

- Forças cortantes: $V_{k, 1}=100 \mathrm{kN}, V_{k, 2}=150 \mathrm{kN}$ e $V_{k, 3}=200 \mathrm{kN}$;

- Modelo de cálculo I: $\theta=45^{\circ}$;

- Modelo de cálculo II: $\theta=30^{\circ}, \theta=35^{\circ}$ e $\theta=45^{\circ}$;

- Espaçamento dos estribos: $s=100 \mathrm{~cm}$.

A partir dos próximos parágrafos, estão descritas as hipóteses de cálculo recomendadas pelos modelos de cálculo I e II, em conformidade com a ABNT NBR 6118 (2014), para a determinação da armadura transversal de vigas em concreto armado. Para isso, estão ilustradas as equações e as considerações para o cálculo dessas armaduras de acordo com os dois modelos utilizados no presente trabalho.

\section{- Modelo de Cálculo I}

O modelo de cálculo I admite que as diagonais de compressão na viga de concreto (bielas de compressão) estão inclinadas em uma angulação correspondente a $\theta=45^{\circ} \mathrm{em}$ 
relação ao eixo longitudinal da viga. Além disso, esse modelo considera uma redução da parcela da força resistente de cálculo $\left(V_{c}\right)$ que independe do valor da força solicitante de cálculo $\left(V_{S d}\right)$. A partir disso, deve-se verificar a segurança do elemento estrutural analisado e a resistência da peça deve ser avaliada com auxílio das Equações 1 a 3.

$$
V_{S d} \leq V_{R d 2,1}
$$

Em que: $V_{S d} \rightarrow$ força cortante solicitante de cálculo; $V_{R d 2,1} \rightarrow$ força cortante resistente de cálculo relativa à ruína das diagonais comprimidas de concreto.

$$
V_{R d 2,1}=0,27 \times \alpha_{V 2} \times f_{c d} \times b_{w} \times d
$$

(Equação 2)

Sendo: $f_{c d} \rightarrow$ resistência de cálculo do concreto a compressão; $b_{w} \rightarrow$ base da viga; $d \rightarrow$ altura útil da viga.

$$
\alpha_{V 2}=1-\frac{f_{c k}}{250}
$$

Em que: $f_{c k} \rightarrow$ resistência característica do concreto a compressão.

Após o cálculo do $V_{S d}$ e $V_{R d 2,1}$, deve-se analisar se a verificação inicial será atendida (Equação 1). Se for, não há perigo de esmagamento das diagonais comprimidas de concreto. Caso a condição expressa pela Equação 1 não seja atendida, deve-se redimensionar a peça ou aumentar a resistência característica do concreto à compressão. Seguindo o procedimento, calcula-se a força cortante a ser resistida pela armadura transversal (Equações 4 a 7).

$$
V_{S W}=V_{S d}-V_{c}
$$

(Equação 4)

Em que: $V_{c} \rightarrow$ parcela de força cortante absorvida por mecanismos complementares ao de treliça.

$$
V_{c}=0,6 \times f_{c t d} \times b_{w} \times d
$$

Sendo: $f_{c t d} \rightarrow$ valor de cálculo da resistência à tração do concreto.

$$
f_{c t d}=0,15 \times\left(f_{c k^{\frac{2}{3}}}\right)
$$


Vale salientar que, se o valor da força cortante resistida pela armadura transversal apresentar valor negativo, significa que o concreto por si só resiste ao cisalhamento, e nesse caso, o estribo mínimo pode ser adotado para a peça. Além disso, a ABNT NBR 6118 (2014) também determina que a força cortante resistida pela armadura transversal pode ser determinada pela Equação 7.

$$
V_{s w}=\frac{A_{s w} \times 0,9 \times d \times f_{y w d} \times(\operatorname{sen} \alpha+\cos \alpha)}{s}
$$

Em que: $A_{S W} \rightarrow$ área total da seção transversal dos estribos; $s \rightarrow$ espaçamento entre os estribos, medido segundo o eixo longitudinal da peça; $\mathrm{f}_{\mathrm{ywd}} \rightarrow$ tensão na armadura transversal passiva, limitada ao valor $\mathrm{f}_{\mathrm{yd}}$ no caso de estribos e a $70 \%$ desse valor no caso de barras dobradas, não se tomando, para ambos os casos, valores superiores a $435 \mathrm{MPa}$; $\alpha \rightarrow$ ângulo de inclinação da armadura transversal em relação ao eixo longitudinal do elemento estrutural, podendo-se tomar $45^{\circ} \leq \alpha \leq 90^{\circ}$.

Por fim, após os procedimentos ilustrados anteriormente, com a determinação da força cortante resistente de cálculo relativa à ruína das diagonais comprimidas de concreto $\left(V_{R d 2}\right)$ e da força cortante resistida pela armadura transversal $\left(V_{s w}\right)$, respectivamente, pode-se calcular a área de aço total da seção transversal dos estribos, que se dá por meio da Equação 8.

$$
\frac{A_{s w}}{s}=\frac{V_{s w}}{0,9 \times d \times f_{y w d} \times(\operatorname{sen} \alpha+\cos \alpha)}
$$

\section{- Modelo de Cálculo II}

Para o modelo de cálculo II, o ângulo das diagonais de compressão do concreto, em relação ao eixo longitudinal da viga, pode variar de acordo com a condição correspondente a $30^{\circ} \leq \theta \leq 45^{\circ}$. De forma análoga ao modelo de cálculo I, a verificação da compressão das diagonais do concreto é obtida por meio da Equação 1. Porém, para esse modelo, calcula-se o $V_{R d 2}$ através da Equação 9. Ademais, para esse modelo, a parcela complementar $V_{c}$ sofre redução à medida que o valor de $V_{s d}$ aumenta.

$$
V_{R d 2,2}=0,54 \times \alpha_{V 2} \times f_{c d} \times b_{w} \times d \times \operatorname{sen}^{2} \theta \times(\operatorname{cotg} \alpha+\operatorname{cotg} \theta)(\text { Equação } 9)
$$


Atendida a condição imposta na Equação 1, segue-se para determinar o valor de $V_{S W}$, onde os cálculos foram análogos aos utilizados nas Equações 3, 4 e 5 para o Modelo de Cálculo I. Porém, para o Modelo II, quanto maior for a força $V_{S d}$, menor será a parcela $V_{c}$. Se $V_{s d} \geq V_{c}$, para que a armadura transversal resista a uma parcela da força cortante, haverá um $V_{c 1}$ menor que $V_{c}$ e que corresponderá ao novo valor da força cortante absorvida por elementos complementares da treliça. Então, o valor do $V_{c 1}$ pode ser obtido por meio da Equação 10 e o valor do $V_{S W}$ através da Equação 11.

$$
\begin{gathered}
V_{c 1}=V_{c} \times \frac{V_{R d 2}-V_{S d}}{V_{R d 2}-V_{c}} \\
V_{S W}=V_{S d}-V_{c 1}
\end{gathered}
$$

A partir disso, de acordo com a determinação da força cortante resistente de cálculo relativa à ruína das diagonais comprimidas de concreto $\left(V_{R d 2}\right)$, da parcela de força cortante absorvida por mecanismos complementares ao de treliça $\left(V_{c}\right)$, da parcela da força $V_{c 1}$ a ser reduzida e da força cortante resistida pela armadura transversal $\left(V_{S w}\right)$, respectivamente, pode-se realizar o cálculo da área de aço da armadura transversal, admitindo o espaçamento entre os estribos igual a 100 centímetros, a partir da Equação 12.

$$
\frac{A_{s w}}{s}=\frac{V_{s w}}{0,9 \times d \times f_{y d} \times(\operatorname{cotg} \alpha+\operatorname{cotg} \theta) \times \operatorname{sen} \alpha}
$$

Ademais, vale salientar que, tanto para o modelo I como para o modelo II, as áreas de aço calculadas devem respeitar o valor da área de aço mínima, que pode ser calculada através das Equações 13 e 14. Esse valor é necessário porque vai ser utilizado no comparativo das armaduras transversais calculadas para avaliar o modelo de cálculo que exige uma menor área de aço.

$$
\begin{gathered}
\frac{A_{s w, \min }}{s} \geq 0,2 \frac{f_{c t m}}{f_{y w k}} \times \operatorname{sen} \alpha \times b_{w} \\
f_{c t m}=0,30 \times\left(f_{c k^{\frac{2}{3}}}\right)
\end{gathered}
$$

Sendo: $\mathrm{f}_{\mathrm{ywk}} \rightarrow$ valor característico da resistência ao escoamento do aço da armadura transversal. 
Em síntese e de acordo com as equações mostradas anteriormente, o primeiro comparativo realizado foi em relação a força solicitante da estrutura $\left(V_{S d}\right)$ com a força resistente relativa à ruína das diagonais comprimidas de concreto $\left(V_{R d 2}\right)$. O próximo comparativo foi relacionado a força solicitante de cálculo $\left(V_{S d}\right)$ com a força cortante absorvida por mecanismos complementares da treliça $\left(V_{c}\right)$. Dando prosseguimento, verificou-se a relação da força solicitante de cálculo $\left(V_{S d}\right)$ com a parcela de redução da força $\left(V_{c 1}\right)$ e, por fim, fez-se uma avaliação a partir da área de aço (armadura transversal) do modelo de cálculo mais econômico.

\section{RESULTADOS E DISCUSSÕES}

Os primeiros cálculos realizados foram em relação à verificação ao esmagamento das bielas de concreto de acordo com cada modelo de cálculo, para os três valores da força cortante máxima e as duas seções transversais. Em conformidade com o modelo de cálculo I, utilizou-se o $\theta=45^{\circ}$ e, para o modelo II, os ângulos escolhidos foram $\theta=30^{\circ}$, $\theta=35^{\circ}$ e $\theta=45^{\circ}$. Para cada uma das forças e de acordo com os ângulos, foram realizadas essas verificações e esses valores estão expostos na Tabela 1.

Tabela 1 - Verificação ao esmagamento das bielas de concreto.

\begin{tabular}{|c|c|c|c|c|c|c|}
\hline $\begin{array}{c}\text { Seção } \\
(\mathbf{c m})\end{array}$ & $\begin{array}{l}\text { Modelo de } \\
\text { Cálculo }\end{array}$ & $\boldsymbol{\theta}\left({ }^{\circ}\right)$ & $\begin{array}{c}V_{k} \\
(\mathbf{K N})\end{array}$ & $\begin{array}{c}V_{s d} \\
(\mathbf{K N})\end{array}$ & $V_{R d 2}(\mathrm{KN})$ & Verificação \\
\hline \multirow{10}{*}{$20 \times 60$} & \multirow{3}{*}{ I } & \multirow{3}{*}{45} & 100 & 140 & \multirow{3}{*}{485,74} & Sem esmagar \\
\hline & & & 150 & 210 & & Sem esmagar \\
\hline & & & 200 & 280 & & Sem esmagar \\
\hline & \multirow{7}{*}{ II } & \multirow{3}{*}{30} & 100 & 140 & \multirow{3}{*}{420,66} & Sem esmagar \\
\hline & & & 150 & 210 & & Sem esmagar \\
\hline & & & 200 & 280 & & Sem esmagar \\
\hline & & \multirow{3}{*}{35} & 100 & 140 & \multirow{3}{*}{456,45} & Sem esmagar \\
\hline & & & 150 & 210 & & Sem esmagar \\
\hline & & & 200 & 280 & & Sem esmagar \\
\hline & & 45 & 100 & 140 & 485,74 & Sem esmagar \\
\hline
\end{tabular}




\begin{tabular}{|c|c|c|c|c|c|c|}
\hline & & & 150 & \multicolumn{2}{|l|}{210} & \multirow{2}{*}{$\begin{array}{l}\text { Sem esmaga } \\
\text { Sem esmaga }\end{array}$} \\
\hline & & & 200 & 280 & & \\
\hline \multirow{12}{*}{$20 \times 70$} & & & 100 & 140 & & Sem esmagar \\
\hline & I & 45 & 150 & 210 & 575,53 & Sem esmagar \\
\hline & & & 200 & 280 & & Sem esmagar \\
\hline & \multirow{9}{*}{ II } & & 100 & 140 & & Sem esmagar \\
\hline & & 30 & 150 & 210 & 495,82 & Sem esmagar \\
\hline & & & 200 & 280 & & Sem esmagar \\
\hline & & & 100 & 140 & & Sem esmagar \\
\hline & & 35 & 150 & 210 & 538,00 & Sem esmagar \\
\hline & & & 200 & 280 & & Sem esmagar \\
\hline & & & 100 & 140 & & Sem esmagar \\
\hline & & 45 & 150 & 210 & 572,56 & Sem esmagar \\
\hline & & & 200 & 280 & & Sem esmagar \\
\hline
\end{tabular}

Fonte: Os Autores (2021).

A partir dos resultados dispostos na Tabela 1, pode-se observar que os valores encontrados respeitam o intervalo estabelecido na metodologia, mostrando que a força solicitante da estrutura $\left(V_{S d}\right)$ é menor que a força resistente relativa à ruína das diagonais comprimidas de concreto $\left(V_{R d 2}\right)$, o que significa que, para todos os resultados obtidos, não houve o esmagamento das bielas de compressão. Ademais, ainda analisando os valores ilustrados na mesma Tabela, percebe-se que, à medida que houve o aumento da seção transversal, para os mesmos valores de angulação, ocorreu um aumento do $V_{R d 2}$, o que proporcionou uma melhor resistência para a peça analisada e propiciou uma maior margem de segurança para a análise desenvolvida neste trabalho.

Ao que refere-se ao comparativo da força solicitante da estrutura com os valores obtidos da força resistente relativa à ruína das diagonais comprimidas de concreto, verifica-se que, o valor de $V_{S d}$ que mais se assemelhou ao $V_{R d 2}$ foi no caso da seção de $20 x 60 \mathrm{~cm}$, modelo de cálculo II, com um ângulo de inclinação das bielas de concreto em relação ao eixo longitudinal da viga referente a $30^{\circ}$, que apresenta força cortante solicitante de cálculo equivalente a $140 \mathrm{kN}$. De maneira análoga, o valor de $V_{S d}$ que mais 
se distanciou ao $V_{R d 2}$ foi no caso da seção de $20 \times 70 \mathrm{~cm}$, modelo de cálculo I, com um ângulo de inclinação das bielas de concreto em relação ao eixo longitudinal da viga referente a $45^{\circ}$, que apresenta força cortante solicitante de cálculo equivalente a $140 \mathrm{kN}$.

De modo geral, observa-se por meio dos comparativos anteriores que, mesmo nas situações em que o $V_{S d}$ mais se assemelhou ao $V_{R d 2}$, a diferença entre esses dois parâmetros ainda é considerável, o que possibilita uma melhor segurança para a peça analisada, como mencionado anteriormente. Sendo assim, em atendimento à condição de segurança, foi dado prosseguimento a análise referente ao dimensionamento das armaduras transversais. Para isso, determinou-se a força cortante absorvida pelos mecanismos complementares da treliça $\left(V_{c}\right)$ e depois a parcela da força cortante resistida pelos estribos $\left(V_{s w}\right)$, de acordo com as Equações 4 e 5 . Esses valores estão indicados na Tabela 2.

Tabela 2 - Valores da força cortante resistida pelos estribos.

\begin{tabular}{|c|c|c|c|c|c|c|}
\hline Seção (cm) & $\begin{array}{l}\text { Modelo de } \\
\text { Cálculo }\end{array}$ & $\boldsymbol{\theta}\left({ }^{\circ}\right)$ & $\begin{array}{c}V_{k} \\
(\mathbf{K N})\end{array}$ & $\begin{array}{c}V_{s d} \\
(\mathbf{K N})\end{array}$ & $V_{c}(\mathbf{K N})$ & $V_{s w}(\mathrm{KN})$ \\
\hline \multirow{12}{*}{$20 \times 60$} & \multirow{3}{*}{ I } & & 100 & 140 & & 53,86 \\
\hline & & 45 & 150 & 210 & \multirow{2}{*}{86,14} & 123,86 \\
\hline & & & 200 & 280 & & 193,86 \\
\hline & & \multirow{3}{*}{30} & 100 & 140 & & 67,73 \\
\hline & & & 150 & 210 & \multirow{2}{*}{86,14} & 155,76 \\
\hline & & & 200 & 280 & & 243,78 \\
\hline & \multirow{6}{*}{ II } & & 100 & 140 & & 66,39 \\
\hline & & 35 & 150 & 210 & \multirow{2}{*}{86,14} & 152,67 \\
\hline & & & 200 & 280 & & 238,96 \\
\hline & & \multirow{3}{*}{45} & 100 & 140 & & 65,47 \\
\hline & & & 150 & 210 & \multirow{2}{*}{86,14} & 150,56 \\
\hline & & & 200 & 280 & & 235,65 \\
\hline \multirow{2}{*}{$20 \times 70$} & \multirow[b]{2}{*}{ I } & \multirow{2}{*}{45} & 100 & 140 & \multirow{2}{*}{101,53} & 38,47 \\
\hline & & & 150 & 210 & & 108,47 \\
\hline
\end{tabular}




\begin{tabular}{|c|c|c|c|c|c|}
\hline & & 200 & 280 & & 178,48 \\
\hline & & 100 & 140 & & 48,38 \\
\hline & 30 & 150 & 210 & 101,53 & 136,40 \\
\hline & & 200 & 280 & & 224,43 \\
\hline & & 100 & 140 & & 47,42 \\
\hline \multirow[t]{5}{*}{ II } & 35 & 150 & 210 & 101,53 & 133,71 \\
\hline & & 200 & 280 & & 219,99 \\
\hline & & 100 & 140 & & 46,77 \\
\hline & 45 & 150 & 210 & 101,53 & 131,86 \\
\hline & & 200 & 280 & & 216,95 \\
\hline
\end{tabular}

Fonte: Os Autores (2021).

Por meio da Tabela 2, observa-se que, para todas as situações analisadas, a força solicitante de cálculo $\left(V_{S d}\right)$ é maior que a força cortante absorvida por mecanismos complementares da treliça $\left(V_{c}\right)$. Quando isso acontece, pode-se determinar um novo valor para o $V_{c}$, como foi dito na metodologia, e isso deve ser feito para que uma parcela da força cortante possa ser absorvida por elementos complementares da treliça. Ademais, pode-se fazer essa análise porque admite-se também uma redução no valor solicitante de cálculo, em função dos mecanismos complementares aos da treliça citados anteriormente, sendo essa redução constante no modelo I para qualquer valor de solicitação, enquanto que no modelo II essa redução é dependente do valor solicitante de cálculo.

A partir disso, para o modelo de cálculo I, a parcela complementar $V_{c}$ tem valor constante independente de $V_{s d}$, então, para esse modelo, o valor de $V_{c}$ permanece o mesmo e não precisa ser recalculado, como já mencionado. No entanto, para o modelo II, este valor é recalculado e corresponde a um valor $V_{c 1}$ menor que $V_{c}$ e, então, recalcula-se também o $V_{s w}$, já que ele depende do $V_{c 1}$. Esses valores estão apresentados na Tabela 3 . 
Tabela 3 - Valores recalculados da força cortante resistida pelos estribos.

\begin{tabular}{|c|c|c|c|c|c|c|}
\hline $\begin{array}{l}\text { Seção } \\
(\mathrm{cm})\end{array}$ & $\begin{array}{l}\text { Modelo de } \\
\text { Cálculo }\end{array}$ & $\boldsymbol{\theta}\left({ }^{\circ}\right)$ & $\begin{array}{c}V_{s d} \\
(\mathbf{k N})\end{array}$ & $V_{c}(\mathrm{kN})$ & $V_{c 1}(\mathrm{kN})$ & $\begin{array}{c}V_{\text {sw,rec }} \\
(\mathbf{k N})\end{array}$ \\
\hline \multirow{9}{*}{$20 \times 60$} & \multirow{9}{*}{ II } & & 140 & & 72,27 & 67,73 \\
\hline & & 30 & 210 & 86,14 & 54,24 & 155,76 \\
\hline & & & 280 & & 36,22 & 243,78 \\
\hline & & & 140 & & 73,01 & 66,39 \\
\hline & & 35 & 210 & 86,14 & 57,33 & 152,67 \\
\hline & & & 280 & & 41,04 & 238,96 \\
\hline & & & 140 & & 74,53 & 65,47 \\
\hline & & 45 & 210 & 86,14 & 59,44 & 150,56 \\
\hline & & & 280 & & 44,35 & 235,67 \\
\hline \multirow{9}{*}{$20 \times 70$} & \multirow{9}{*}{ II } & & 140 & & 91,62 & 48,38 \\
\hline & & 30 & 210 & 101,53 & 73,60 & 136,40 \\
\hline & & & 280 & & 55,57 & 224,43 \\
\hline & & & 140 & & 92,58 & 47,42 \\
\hline & & 35 & 210 & 101,53 & 76,29 & 133,70 \\
\hline & & & 280 & & 60,01 & 219,99 \\
\hline & & & 140 & & 93,23 & 46,77 \\
\hline & & 45 & 210 & 101,53 & 78,14 & 131,86 \\
\hline & & & 280 & & 63,06 & 216,95 \\
\hline
\end{tabular}

Fonte: Os Autores (2021).

Analisando os valores ilustrados na Tabela 3, observa-se que, para a mesma angulação analisada, à medida que a solicitação vai aumentando $\left(V_{S d}\right)$, a parcela da força $V_{c 1}$ a ser reduzida vai diminuindo. Isso significa que, quanto mais essa parcela de redução vai diminuindo, maior tem que ser a força cortante resistida pela armadura transversal $\left(V_{S w}\right)$, evidenciando que a resistência da estrutura deve aumentar conforme a solicitação vai sendo incrementada, comportamento já esperado e também observado nos trabalhos 
de Barros e Giongo (2008), Samora (2015), Carvalho (2016) e Pereira, Lima e Correia (2021a).

Em síntese, para o modelo de cálculo II, quanto maior for a solicitação, menor vai ser a parcela $V_{c 1}$ a ser reduzida, atingindo valor zero quando a solicitação for igual à capacidade resistente da biela de compressão. Ademais, pode-se verificar que esse comportamento ocorreu de maneira similar para as duas seções transversais analisadas, o que indica que esse desempenho independe da geometria da estrutura e é influenciado diretamente em conformidade com a força cortante solicitante de cálculo.

Após a determinação da força cortante a ser resistida pela armadura transversal (Tabelas 2 e 3), para os modelos de cálculo I e II, respectivamente, foram calculadas as armaduras transversais necessárias para os dois modelos mencionados. Os resultados obtidos estão dispostos na Tabela 4 e o comparativo do modelo II em relação ao modelo I foi realizado (Observação: A área de aço mínima calculada para a peça analisada foi de $\left.2,05 \mathrm{~cm}^{2} / \mathrm{m}\right)$.

Tabela 4 - Resultados da armadura transversal.

\begin{tabular}{|c|c|c|c|c|c|}
\hline Seção $(\mathrm{cm})$ & $V_{s d}(\mathrm{kN})$ & $\begin{array}{l}\text { Modelo de } \\
\text { Cálculo }\end{array}$ & $\boldsymbol{\theta}\left(^{\circ}\right)$ & $\begin{array}{c}A_{S} \\
\left(\mathrm{~cm}^{2} \cdot \mathrm{m}^{-1}\right)\end{array}$ & $\Delta(\%)$ \\
\hline \multirow{12}{*}{$20 \times 60$} & \multirow{4}{*}{140} & I & 45 & 2,47 & \\
\hline & & \multirow{3}{*}{ II } & 30 & 2,05 & $-17,53$ \\
\hline & & & 35 & 2,12 & $-14,17$ \\
\hline & & & 45 & 2,99 & 21,05 \\
\hline & \multirow{4}{*}{210} & I & 45 & 5,67 & \\
\hline & & \multirow{3}{*}{ II } & 30 & 4,11 & $-27,51$ \\
\hline & & & 35 & 4,88 & $-13,93$ \\
\hline & & & 45 & 6,87 & 21,16 \\
\hline & \multirow{4}{*}{280} & I & 45 & 8,88 & \\
\hline & & \multirow{3}{*}{ II } & 30 & 5,45 & $-38,63$ \\
\hline & & & 35 & 6,48 & $-27,03$ \\
\hline & & & 45 & 9,13 & 2,82 \\
\hline
\end{tabular}




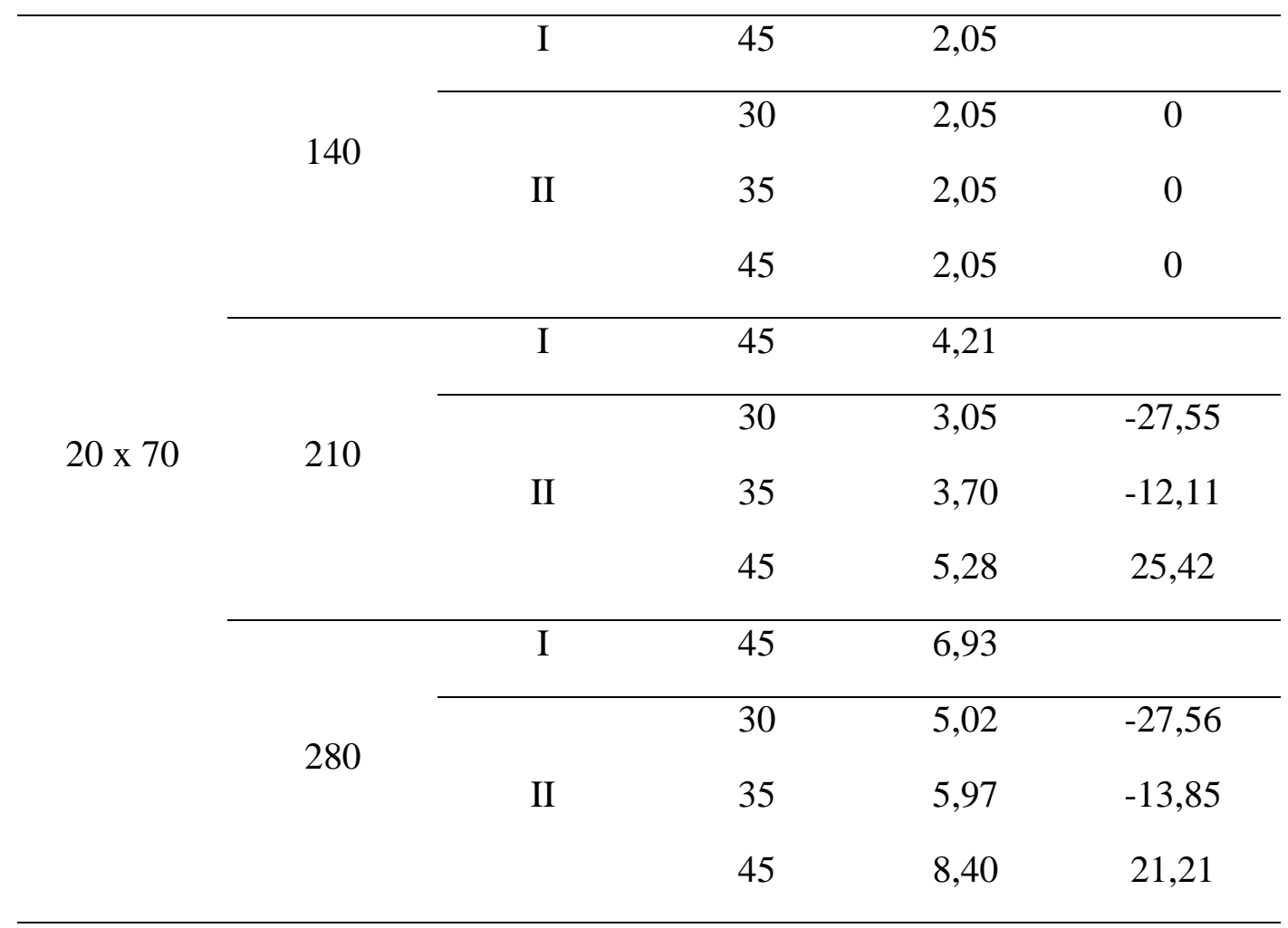

Fonte: Os Autores (2021).

Com os resultados dispostos na Tabela 4, é possível perceber que houve uma variação no cálculo das armaduras transversais pelos dois métodos avaliados. Pode-se observar, em relação ao modelo de cálculo II, que à medida que se aumenta o valor de $\theta$, para a mesma intensidade de força solicitante de cálculo, o valor da área de aço para o estribo também aumenta. Ademais, como mencionado anteriormente, a variação mostrada na tabela correspondente a dispersão do modelo II em relação ao modelo I.

Ainda observou-se que, para a seção 20x60 cm, para os ângulos de $\theta=30^{\circ} \mathrm{e}$ $\theta=35^{\circ}$ utilizados no modelo II, em relação ao ângulo de $\theta=45^{\circ}$ para o modelo I e $V_{S d}=140 \mathrm{kN}$, há uma economia na área de aço que fica em torno de $17 \%$ e $14 \%$, respectivamente, se utilizado o modelo II; para os ângulos de $\theta=30^{\circ}$ e $\theta=35^{\circ}$ utilizados no modelo II, em relação ao ângulo de $\theta=45^{\circ}$ para o modelo I e $V_{S d}=210 \mathrm{kN}$, há uma economia na área de aço que fíca em torno de $27 \%$ e $13 \%$, respectivamente, se utilizado o modelo II; para os ângulos de $\theta=30^{\circ}$ e $\theta=35^{\circ}$ utilizados no modelo II, em relação ao ângulo de $\theta=45^{\circ}$ para o modelo I e $V_{S d}=280 \mathrm{kN}$, há uma economia na área de aço que fica em torno de $38 \%$ e $27 \%$, respectivamente, se utilizado o modelo II. 
De modo geral, verifica-se que, para $\theta=30^{\circ}$ e $\theta=35^{\circ}$, o modelo de cálculo II torna-se mais econômico e, para $\theta=45^{\circ}$, o modelo de cálculo I torna-se mais econômico. A partir disso, pode-se verificar que o comportamento descrito também é verificado para a seção transversal $20 \times 70 \mathrm{~cm}$, o que mostra que, para o modelo de cálculo II, a armadura transversal resulta em valores maiores para o $\theta=45^{\circ}$, aumentando na ordem de $20 \% \mathrm{em}$ relação ao modelo I. Vale salientar que, para a análise da seção $20 \times 70 \mathrm{~cm}$, tanto o modelo de cálculo I como o modelo II apresentam a utilização da área de aço mínima para um esforço cortante de $V_{S d}=140 \mathrm{kN}$, sendo por isso apresentada uma variação nula.

Sendo assim, esses resultados confirmam os estudos de Samora (2015) e Meller e Vargas (2016), que afirmam que, ao adotar $\theta<39^{\circ}$, os valores da área da armadura transversal para o modelo II serão mais econômicos e para $\theta=45^{\circ}$, o modelo I torna-se mais econômico. Ainda, para complementar o estudo, Barros e Giongo (2008) afirmam que, para valores de ângulos situados no intervalo $30^{\circ} \leq \theta \leq 39^{\circ}$, a área da armadura transversal pelo modelo de cálculo II resulta em valores menores, obtendo seu valor mínimo para $\theta=30^{\circ}$. Ademais, é interessante notar que, também nos valores de armadura transversal, a redução percentual na área de aço é similar para qualquer problema, não sendo influenciada nem pela intensidade das ações, nem pela geometria do problema.

A Figura 1 ilustra os dados compilados dessa análise, de forma que a visualização desses resultados se torne mais clara, lembrando que não houve comparação para a análise da seção $20 \times 70 \mathrm{~cm}$ utilizando a força $V_{S d}=140 \mathrm{kN}$, já que nessa situação todos os elementos se apresentaram com a área de aço mínima. 
Figura 1 - Comparação dos resultados obtidos (em relação à área de aço).

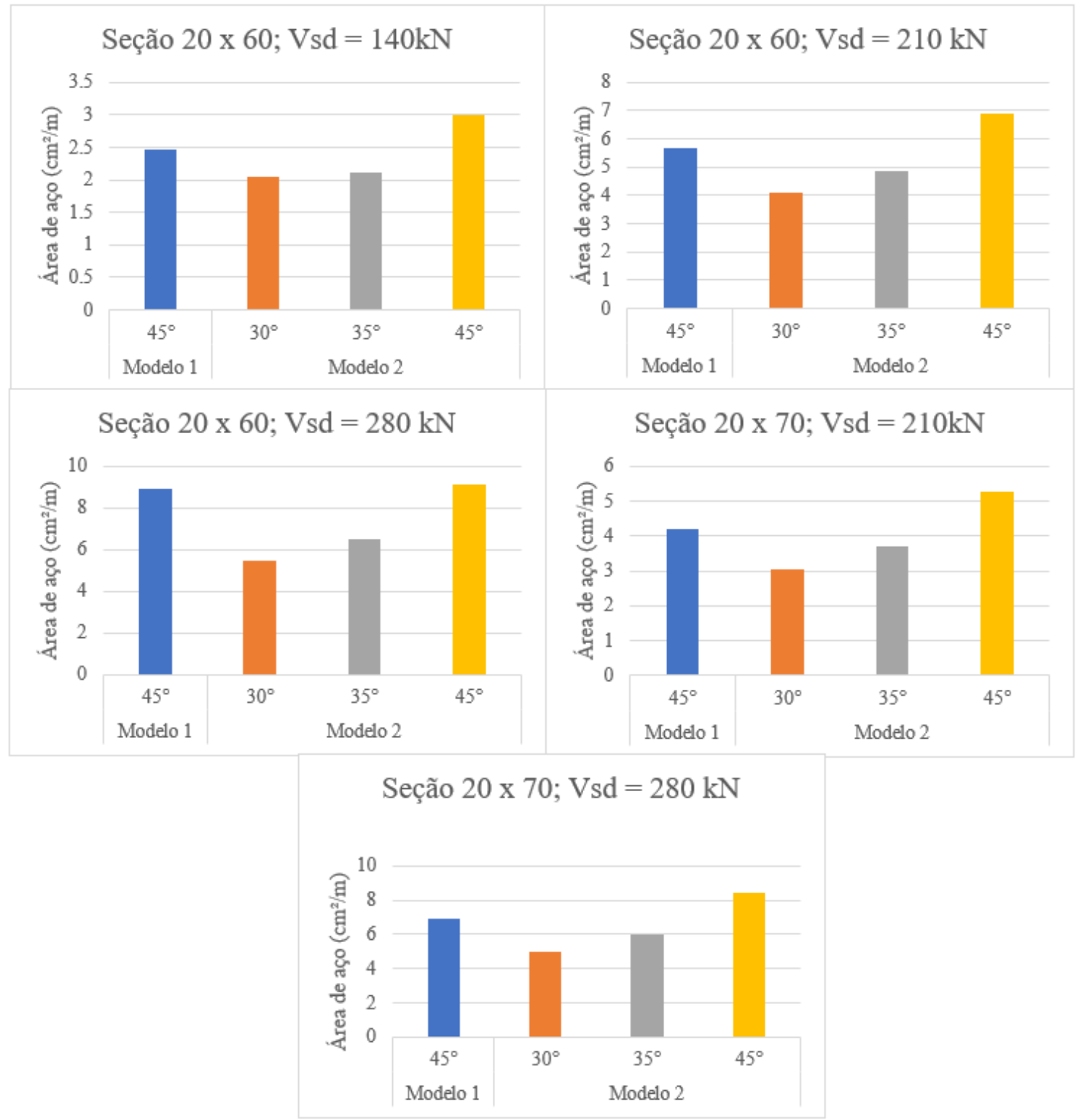

Fonte: Os Autores (2021).

Através da Figura 1, em resumo, pode-se notar que inicialmente o ângulo $\theta=$ $30^{\circ}$, para o segundo modelo de cálculo, é a opção mais vantajosa economicamente por apresentar uma redução na área de aço em torno de $12 \%$ a $38 \%$, se comparado com o primeiro modelo. Em contrapartida, esse ângulo apresenta menores valores de $V_{R d 2}$ (Tabela 1), se comparado com os outros ângulos analisados, o que pode acarretar o não atendimento da verificação da segurança em relação ao esmagamento das bielas de concreto. Dessa forma, portanto, verifica-se que para $\theta=45^{\circ}$, o modelo I foi mais econômico que o modelo II, como também evidenciado nos trabalhos de Pereira, Lima e Correia (2021b). 


\section{CONCLUSÕES}

De acordo com a investigação feita, percebeu-se que o cálculo da armadura transversal de vigas, seguindo os métodos propostos pela ABNT NBR 6118 (2014), apesar de serem baseados na mesma hipótese, apresentam diferentes condições de cálculo e, por consequência, denotam valores distintos de armaduras transversais. Assim sendo, os procedimentos apresentam divergências quanto ao valor encontrado para área de aço necessária e isto pode ser explicado com base na angulação de $\theta$, pois a depender do valor e metodologia empregada, o valor de área de aço pode variar.

De forma geral, em relação ao modelo de cálculo II, à medida que se aumenta o valor de $\theta$, para a mesma intensidade de força solicitante de cálculo, o valor da área de aço para armadura transversal também aumenta. Ademais, o modelo II se mostrou mais econômico que o modelo I para fissuras com inclinação entre $30^{\circ}$ e $35^{\circ}$, no entanto, para fissuras com inclinação igual a $45^{\circ}$, o modelo I foi que apresentou a maior economia de aço, o que evidencia as diferenças relatadas no parágrafo anterior.

Diante disso, percebe-se que, ao adotar $\theta<45^{\circ}$, os valores da área da armadura transversal para o modelo II são mais econômicos e, para $\theta=45^{\circ}$, o modelo I torna-se mais econômico, independentemente da geometria adotada, com ambos os modelos atendendo aos padrões de segurança. Sendo assim, pode-se dizer que o presente trabalho alcançou as metas sugeridas inicialmente, onde foi realizado um estudo comparativo entre dois modelos distintos para o cálculo da armadura transversal de vigas, os modelos I e II da ABNT NBR 6118 (2014), onde verificou-se que a depender da angulação adotada, entre as bielas de concreto e o eixo longitudinal da viga, o valor da armadura transversal é modificada e, considerando as mesmas condições de angulação, o modelo I torna-se o modelo de cálculo mais econômico.

\section{REFERÊNCIAS}

ARAÚJO, J. M. Estruturas de concreto: a resistência à compressão e critérios de ruptura para o concreto. Rio Grande: Dunas, 2001, $\mathrm{n}^{\circ} 1$.

Curso de Concreto Armado. 2 Ed. Rio Grande: Dunas, 2010, v.2. 
ASSOCIAÇÃO BRASILEIRA DE NORMAS TÉCNICAS. ABNT NBR 6118: Projeto de estruturas de concreto armado - procedimento. Rio de Janeiro, 2014.

ALMEIDA, L. C. Dimensionamento de vigas de concreto armado ao esforço cortante. Universidade Estadual Paulista: São Paulo, 2004.

BARROS, R.; GIONGO, J. S. Cálculo da área da armadura transversal em elementos lineares de concreto armado submetidas à ação de força cortante: análise comparativa entre os Modelos I e II da NBR 6118:2003. In: Anais do $50^{\circ}$ Congresso Brasileiro do Concreto - IBRACON. Salvador, 2008.

BASTOS, P. S. S. Dimensionamento de vigas de concreto armado à força cortante. Universidade Estadual Paulista: São Paulo, 2015.

Paulo, 2017.

Torção em vigas de concreto armado. Universidade Estadual Paulista: São

BRANDÃO, A. M. S. Qualidade e durabilidade das estruturas de concreto armado: aspectos relativos ao projeto. Universidade de São Paulo: São Carlos, 1998.

CARVALHO, R. C.; FIGUEIREDO FILHO, J. R. Cálculo e detalhamento de estruturas usuais de concreto armado segundo a NBR 6118:2014. 4. ed. São Carlos: EdUFSCar, 2016.

CARVALHO, A. C. F. Análise comparativa do dimensionamento de vigas de concreto armado ao cisalhamento por meio dos modelos de cálculo I e II da NBR 6118:2014. Faculdade Pio Décimo: Aracaju, 2016.

COELHO NETO, W. Influência dos ângulos de biela e tirante no volume de armadura transversal de vigas. Trabalho de Conclusão de Curso. Universidade Tecnológica Federal do Paraná, 2017.

FÉDÉRATION INTERNATIONALE DU BÉTON - fib. fib model code for concrete structures 2010. Switzerland, 2013.

FUSCO, P. B. Técnica de armar as estruturas de concreto. São Paulo, Ed. Pini, 2000.

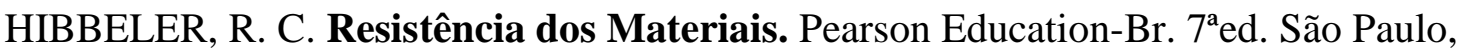
2010.

ISHIHARA, R. Avaliação de ensaios experimentais de cisalhamento em vigas de concreto armado sem armadura transversal à luz das normas. 2017.

PEREIRA, V. B. S.; LIMA, I. E. P.; CORREIA, V. C. Análise Comportamental de Vigas em Concreto Armado Através da Determinação da Armadura Transversal. In: $62^{\circ}$ Congresso Brasileiro do Concreto, 2021. Anais do $62^{\circ}$ IBRACON, 2021a. 
PEREIRA, V. B. S.; LIMA, I. E. P.; CORREIA, V. C. Análise da Armadura Transversal em Vigas de Concreto Armado. In: XII Congresso de Engenharia, Ciência e Tecnologia, 2021, Maceió - AL. Anais do XII CONECTE, 2021 b.

MARCELLI, M. Sinistros na construção civil: Causas e soluções para danos e prejuízos em obras. 3 ed. São Carlos/SP: edPini, 2007.

MELLER, B. J.; VARGAS, A. Análise dos modelos de cálculo propostos pela NBR 6118:2014 para o dimensionamento de estribos em vigas de concreto armado. Universidade do Extremo Sul Catarinense: Santa Catarina, 2016.

PINHEIRO, L. M. Fundamentos do Concreto e Projeto de Edifícios. Universidade de São Paulo: São Carlos, 2007.

PINHEIRO, L. M.; MUZARDO, C. D.; SANTOS, S, P. Estruturas de concreto: cisalhamento em vigas. Universidade de São Paulo: São Carlos, 2003.

SADD, M. H. Elasticity: Theory, Application sand Numerics. Second Edition. London, Academic Press, 2009.

SAMORA, M. S. Avaliação dos mecanismos resistentes ao cisalhamento em concreto armado sem armadura transversal. 2015.

VITOR, P. D. C. P.; SANTOS, A. C. D.; TRAUTWEIN, L. M. Resistência ao cisalhamento em vigas de concreto armado sem armadura transversal reforçadas com fibras de aço. Ambiente Construído, 18, 255-270. 2018. 